Technical Report CIT-CDS 03-005

\title{
Agreement Problems in Networks with Directed Graphs and Switching Topology
}

\author{
Reza Olfati Saber Richard M. Murray \\ Control and Dynamical Systems \\ California Institute of Technology \\ e-mails:\{olfati,murray\}@cds.caltech.edu
}

February 15, 2003

Submitted to the IEEE Conference on Decision and Control 2003

\begin{abstract}
In this paper, we provide tools for convergence and performance analysis of an agreement protocol for a network of integrator agents with directed information flow. Moreover, we analyze algorithmic robustness of this consensus protocol for the case of a network with mobile nodes and switching topology. We establish a connection between the Fiedler eigenvalue of the graph Laplacian and the performance of this agreement protocol. We demostrate that a class of directed graphs, called balanced graphs, have a crucial role in solving average-consensus problems. Based on the properties of balanced graphs, a group disagreement function (i.e. Lyapunov function) is proposed for convergence analysis of this agreement protocol for networks with directed graphs. This group disagreement function is later used for convergence analysis for the agreement problem in networks with switching topology. We provide simulation results that are consistent with our theoretical results and demonstrate the effectiveness of the proposed analytical tools.
\end{abstract}

\section{Introduction}

Distributed decision-making for coordination of networks of dynamic agents has attracted several researchers in recent years. This is partly due to broad application of multi-agent system is many areas including cooperative control of unmanned air vehicles (UAVs), flocking of birds $[16,18,17]$, schooling for underwater vehicles, distributed sensory networks, attitude alignment of clusters of satellites, and congestion control in communication networks [15].

Agreement problems have a long history in the field of computer science, particularly in automata theory and distributed computation [12]. In many applications involving multi-agent/multivehicle systems, groups of agents need to agree upon certain quantities of interest. Such quantities might or might not be related to the motion of the individual agents. As a result, it is important to address agreement problems in their general form (as far as the inter-agent communication is 
concerned) for networks of dynamic agents with directed information flow under link failure and creation (i.e. variable network topology).

Our main contribution in this paper is to provide convergence, performance, and robustness analysis of an agreement protocol for a network of integrator agents with directed information flow and (perhaps) switching topology.

In the past, a number of researchers have worked in problems that are essentially different forms of agreement problems with differences regarding the types of agent dynamics, the properties of the graphs, and the names of the tasks of interest. In [19, 5, 4], graph Laplacians are used for the task of formation stabilization for groups of agents with linear dynamics. The drawbacks of a pure linear approach to formation stabilization can be summarized as follows: i) collision-avoidance cannot be directly taken into account (or be formulated as part of the problem), ii) performing the rotation of a formation cannot be addressed, and iii) the method has not yet been extended to systems with nonlinear dynamics that are not feedback linearizable. Special cases of this approach are known as leader-follower type architectures and have been widely used by numerous researchers $[13,2,11]$. In $[18,17]$, flocking and self-alignment is analyzed from the point of view of statistical mechanics and a phase transition phenomenon is observed that is equivalent to connectivity of graphs. The work in [10] focuses on attitude alignment for undirected dynamic graphs in which the agents have simple dynamics motivated by the model used in [18]. It is claimed that the connectivity of the graph on average is sufficient for convergence of the heading angles of the agents. In [14], the authors addressed convergence of linear and nonlinear protocols for networks with undirected links in presence or lack of communication time-delays. Theoretically, the linear case of agreement in an undirected network without time-delay is much easier than the analysis for the case of directed graphs in the present paper.

In this paper, we provide convergence analysis of an agreement protocol for a network of integrators with a directed information flow and fixed or switching topology. Our analysis relies on several tools from algebraic graph theory $[1,8]$ and matrix theory [9]. We establish a connection between the performance of this consensus protocol and the Fiedler eigenvalue of graph Laplacian which is also known as the algebraic connectivity of the graph. It turns out that a class of directed graphs called balanced graphs have a crucial role in derivation of an invariant quantity and a Lyapunov function for convergence analysis of average-consensus problems on directed graphs. This Lyapunov function is a measure of group disagreement in the network. We show that a directed graph solves the average-consensus problem using protocol A if and only if it is balanced. Furthermore, we use properties of balanced networks to analyze the convergence of an agreement protocol for networks with switching topology. This variation of the network topology is usually due to link failures or creations in networks with mobile nodes. We introduce a common Lyapunov function that guarantees asymptotic convergence to a consensus in a network with switching information flow. We provide simulation results that demonstrate our theoretical predictions and show the novel analytical tools that we propose are effective.

An outline of this paper is as follows. In Section 2, we provide some background on algebraic graph theory. In Section 3, we present the setup for agreement problems in directed networks with fixed or switching topology. In Section 4, we state some useful results in matrix theory that are later used in this paper. Our main results are given in separate subsections of Section 5. In Section 6, the simulation results are presented. for agreement on four digraphs and average-consensus problem for a network with switching information flow. Finally, in Section 7, we make our concluding remarks. 


\section{Preliminaries: Algebraic Graph Theory}

In this section, we introduce some basic concepts and notation in graph theory that will be used throughout the paper. More information is available in $[8,3]$.

Let $G=(\mathcal{V}, \mathcal{E}, \mathcal{A})$ be a weighted directed graph (or digraph) with $n$ nodes and a weighted adjacency matrix $\mathcal{A}=\left[a_{i j}\right]$ where $a_{i j} \geq 0$ for all $i, j \in \mathcal{I}=\{1,2, \ldots, n\}: i \neq j$ and $a_{i i}=0$ for all $i \in \mathcal{I}$. The set of neighbors of the node $v_{i}$ is denoted by $N_{i}$ and defined as $N_{i}=\left\{j \in \mathcal{I}: a_{i j}>0\right\}$. The in-degree and out-degree of node $v_{i}$ are, respectively, defined as follows:

$$
\operatorname{deg}_{\text {in }}\left(v_{i}\right)=\sum_{j=1}^{n} a_{j i}, \operatorname{deg}_{\text {out }}\left(v_{i}\right)=\sum_{j=1}^{n} a_{i j} .
$$

For an ordinary graph with $\mathcal{A}$ that has binary elements $\operatorname{deg}_{\text {out }}\left(v_{i}\right)=\left|N_{i}\right|$. The degree matrix of $G$ is a diagonal matrix denoted by $\Delta=\left[\Delta_{i j}\right]$ where $\Delta_{i j}=0$ for all $i \neq j$ and $\Delta_{i i}=\operatorname{deg}_{\text {out }}\left(v_{i}\right)$. The (weighted) graph Laplacian matrix associated with $G$ is defined as

$$
L=\mathcal{L}(G)=\Delta-\mathcal{A} .
$$

With a slight misuse of notation, we use $\mathcal{L}(G)=\mathcal{L}(\mathcal{A})$ to denote the Laplacian of graph $G$. By definition, the graph Laplacian has an eigenvector at $\lambda_{1}=0$ and a right eigenvector $w_{r}=\mathbf{1}=$ $(1,1, \ldots, 1)^{T}$ with identical nonzero elements. Furthermore, for a strongly connected digraph $G$ of order $n$, the Laplacian matrix satisfies the following rank condition:

$$
\operatorname{rank}(L)=n-1
$$

A digraph is called strongly connected if and only if any two distinct nodes of the graph can be connected via a path that respects the orientation of the edges of the digraph.

Note. Throughout this paper, we assume all graphs have at least two nodes (i.e. are non-trivial) and there is no cycle of length one (i.e. an edge from a node to itself).

For an undirected graph $G, L$ is symmetric and positive semi-definite. The disagreement function (also referred to as Laplacian potential) associated with $G$ is defined in [14] as follows

$$
\Phi_{G}(x)=x^{T} L x=\frac{1}{2} \sum_{i j \in \mathcal{E}}\left(x_{j}-x_{i}\right)^{2}
$$

where $x_{i}$ denotes the value of node $v_{i}$. The value of a node might represent physical quantities including attitude, position, temperature, voltage, and so on. We say two distinct nodes $v_{i}$ and $v_{j}$ agree if and only if $x_{i}=x_{j}$. Apparently, $\Phi_{G}(x)=0$ if and only if all neighboring nodes in $G$ agree. If in addition, the graph is connected, then all nodes in the graph agree and a consensus is reached. Therefore, $\Phi_{G}(x)$ is a meaningful function that quantifies the group disagreement in a network.

For an undirected graph $G$ that is connected the following well-known property holds [8]:

$$
\min _{\substack{x \neq 0 \\ \mathbf{1}^{T} x=0}} \frac{x^{T} L x}{\|x\|^{2}}=\lambda_{2}(L)
$$

The proof follows from a special case of Courant-Fischer Theorem in [9]. We will later establish a connection between $\lambda_{2}(\hat{L})$, called the Fiedler eigenvalue of $\hat{L}[6,7]$, and the performance of a linear agreement protocol where $\hat{L}$ is closely-related to $L$. 


\section{Agreement Problem on Directed Graphs}

Consider a network of integrators

$$
\dot{x}_{i}=u_{i}, i \in \mathcal{I}, x_{i}, u_{i} \in \mathbb{R}
$$

with information flow (or topology) $G=(\mathcal{V}, \mathcal{E}, \mathcal{A})$.

Remark 1. The main reason behind focusing on an interconected system with nodes that have extremely simple dynamics is that we would like to discuss the main network and communication related aspects of agreement rather than addressing the compelexities that arise from considering agents with nonlinear dynamics and possible input constraints.

The asymptotic agreement problem can be described as follows. Give a protocol that guarantees the state of the network as a whole asymptotically converges to an equilibrium state $x^{*} \in \mathbb{R}^{n}$ with identical elements, i.e. $x_{i}^{*}=x_{j}^{*}=: \alpha$ for all $i, j \in \mathcal{I}, i \neq j$. The element $\alpha$ that determines $x^{*}$ is called the group decision value. An agreement problem in which $\alpha=A v e(x(0))$ is referred to as the average-consensus problem where $A v e(x)=\left(\sum_{i=1}^{n} x_{i}\right) / n$. Further information regarding a formal definition of average-consensus problem is available in [14].

In this paper, our goal is to address convergence and performance issues of linear consensus protocols for a network with directed information flow. Furthermore, we analyze convergence of the distributed agreement algorithms for networks with variable topology, or switching information flow.

We focus on solving the average-consensus problem using the following agreement protocol:

$$
u_{i}(t)=\sum_{j \in N_{i}} a_{i j}\left(x_{j}(t)-x_{i}(t)\right), i \in \mathcal{I}
$$

In distributed average-consensus problem, the objective of each node of the network is to calculate the average of the initial values of all $n$ nodes provided that no node has an edge with all other nodes (unless $n=2$ ) and the network is connected.

Given Protocol (A), the state of the network evolves according to the following linear system

$$
\dot{x}(t)=-L x(t)
$$

where $L=\mathcal{L}(G)$ is the Laplacian induced by the information flow $G$. In a network with variable topology $G$, convergence analysis of Protocol (A) is equivalent to stability analysis for a hybrid system

$$
\dot{x}(t)=-L_{k} x(t), \quad k=s(t)
$$

where $L_{k}=\mathcal{L}\left(G_{k}\right)$ is the Laplacian of $G_{k}, s(t): \mathbb{R} \rightarrow \mathcal{I}_{\Gamma} \subset \mathbb{Z}$ is a switching signal, and $\Gamma \ni G_{k}$ is a finite collection of digraphs (of order $n$ ) with the index set $\mathcal{I}_{\Gamma}$. Later, we will see that $\Gamma$ is a relatively large set for $n \gg 1$. The task of stability analysis for the hybrid system in (7) is rather challenging partly because $q, p \in \mathcal{I}_{\Gamma}, q \neq p$ most likely implies $L_{q} L_{p} \neq L_{q} L_{p}$. Thus, rather simple ways of constructing a common Lyapunov function for this switching system fail. 


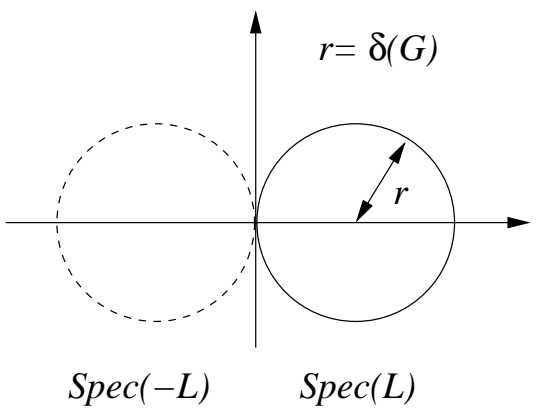

Figure 1: A demonstration of Geršgorin Theorem applied to graph Laplacian.

\section{Matrix Theory and Properties of Graph Laplacians}

The key in the stability analysis of system (6) is in the spectral properties of graph Laplacian. The following result follows from Geršgorin disk theorem [9]:

Proposition 1. (spectral localization) Let $G=(\mathcal{V}, \mathcal{E}, \mathcal{A})$ be a digraph with the Laplacian L. Denote the maximum node out-degree of $G$ by $\delta(G)=\max _{i} \operatorname{deg}_{\text {out }}\left(v_{i}\right)$. Then, all the eigenvalues of $L=$ $\mathcal{L}(G)$ are located in the following disk

$$
D(G)=\{z \in \mathbb{C}:|z-\delta(G)| \leq \delta(G)\}
$$

centered at $z=\delta(G)+0 j$ in the complex plane (see Figure 1). Moreover, the real-part of the eigenvalues of $-L$ are non-positive.

Proof. Based on the Geršgorin disk theorem, all the eigenvalues of $L=\left[l_{i j}\right]$ are located in the union of the following $n$ disks

$$
D_{i}=\left\{z \in \mathbb{C}:\left|z-l_{i i}\right| \leq \sum_{j \in \mathcal{I}, j \neq i}\left|l_{i j}\right|\right\}
$$

But $l_{i i}=\Delta_{i i}$ and

$$
\sum_{j \in \mathcal{I}, j \neq i}\left|l_{i j}\right|=\operatorname{deg}_{\text {out }}\left(v_{i}\right)=\Delta_{i i}
$$

Thus, $D_{i}=\left\{z \in \mathbb{C}:\left|z-\Delta_{i i}\right| \leq \Delta_{i i}\right\}$. On the other hand, all these $n$ disks are contained in the largest disk $D(G)$ with radius $\Delta(G)$. Clearly, all the eigenvalues of $-L$ are located in the disk $D^{\prime}(G)=\{z \in \mathbb{C}:|z+\delta(G)| \leq \delta(G)\}$ that is the mirror image of $D(G)$ with respect to the imaginary axis and the result follows.

The following result guarantees the convergence of Protocol (A) for directed graphs.

Proposition 2. Consider a network of integrators with an information flow $G$ that is a strongly connected digraph. Then, Protocol (A) globally asymptotically solves an agreement problem, i.e. the solution asymptotically converges to an equilibrium $x^{*}$ such that $x_{i}^{*}=x_{j}^{*}$ for all $i, j, i \neq j$.

Proof. Since $G$ is strongly connected, $\operatorname{rank}(L)=n-1$ and $L$ has a zero eigenvalue $\lambda_{1}=0$ with algebraic multiplicity of one. Based on Proposition 1, the rest of the eigenvalues of $L$ have negative 


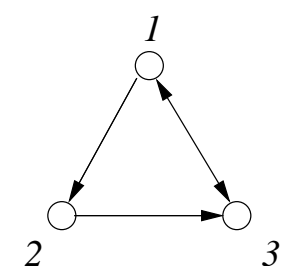

Figure 2: A connected digraph of order 3 that does not solve the average-consensus problem using Protocol (A).

real-parts and therefore the linear system in (6) is stable. On the other hand, any equilibrium $x^{*}$ of (6) is a right eigenvector of $L$ associated with $\lambda_{1}=0$. Since the eigenspace associated with the zero eigenvalue is one-dimensional, there exists an $\alpha \in \mathbb{R}$ such that $x^{*}=\alpha \mathbf{1}$, i.e. $x_{i}^{*}=x_{j}^{*}=\alpha, \forall i, j$.

Keep in mind that Proposition 2 does not guarantee whether the decision value $\alpha$ of each node is equal to Ave $(x(0))$ or not. In other words, Proposition 2 does not address the average-consensus problem.

A sufficient condition for the decision value of each node $\alpha$ to be equal to $\operatorname{Ave}(x(0)$ is that $\sum_{i=1}^{n} u_{i} \equiv 0$. If $G$ is undirected (i.e. $a_{i j}=a_{j i}>0, \forall i, j: a_{i j} \neq 0$ ), automatically the condition $\sum_{i=1}^{n} u_{i}=0, \forall x$ holds and Ave $(x(t))$ is an invariant quantity [14]. However, this property does not hold for a general digraph.

A simple counterexample is a strongly connected digraph of order $n=3$, shown in Figure 2, that is unweighted and has the following sets of vertices and edges:

$$
\mathcal{V}=\{1,2,3\}, \mathcal{E}=\{12,23,31,13\}
$$

For the digraph $G=(\mathcal{V}, \mathcal{E}), \sum_{i=1}^{3} u_{i}=x_{3}-x_{1}$. Thus, if nodes 1 and 3 disagree, the property $\sum_{i=1}^{3} u_{i}=0$ does not hold for all $x$. On the other hand, the reader can verify that for this example

$$
L=\left[\begin{array}{ccc}
2 & -1 & -1 \\
0 & 1 & -1 \\
-1 & 0 & 1
\end{array}\right]
$$

and $x_{i}^{*}=\left[x_{1}(0)+x_{2}(0)+2 x_{3}(0)\right] / 4$ (this is due to Theorem 1 ). This decision value is apparently in the convex hull of all the $x_{i}(0)$ 's but it is different from Ave $(x(0))$ if and only if $x_{1}(0)+x_{2}(0) \neq$ $2 x_{3}(0)$. As a result, for all initial conditions satisfying $x_{1}(0)+x_{2}(0) \neq 2 x_{3}(0)$, Protocol (A) does not solve the average-consensus problem but still all nodes reach an agreement. This motivates us to characterize the class of all digraphs that solve the average-consensus problem.

Before presenting our main result, we need to provide a limit theorem for exponential matrices of the form $\exp (-L t)$. This is because the solution of $(6)$ is given by

$$
x(t)=\exp (-L t) x(0)
$$

and by explicit calculation of $\exp (-L t)$, we can obtain the decision value of each node after reaching consensus for a general digraph. The following theorem is closely related to a famous limit theorem in the theory of non-negative matrices known as Perron-Frobenius Theorem [9] (we discuss the specifics of this relation in an upcoming paper). 
Notation. Following the notation in [9], we denote the set of $m \times n$ real matrices by $M_{m, n}$ and the set of square $n \times n$ matrices by $M_{n}$. Furthermore, throughout this paper, the right and left eigenvectors of the Laplacian $L$ associated with $\lambda_{1}=0$ are denoted by $w_{r}$ and $w_{l}$, respectively.

Theorem 1. Assume $G$ is a strongly connected digraph with Laplacian $L$ satisfying $L w_{r}=0$, $w_{l}^{T} L=0$, and $w_{l}^{T} w_{r}=1$. Then

$$
R=\lim _{t \rightarrow+\infty} \exp (-L t)=w_{r} w_{l}^{T} \in M_{n}
$$

Proof. Let $A=-L$ and let $J$ be the Jordan form associated with $A$, i.e. $A=S J S^{-1}$. We have $\exp (A t)=S \exp (J t) S^{-1}$ and as $t \rightarrow+\infty, \exp (J t)$ converges to a matrix $Q=\left[q_{i j}\right]$ with a single nonzero element $q_{11}=1$. The fact that other blocks in the diagonal of $\exp (J t)$ vanish is due to the property that $\operatorname{Re}\left(\lambda_{k}(A)\right)<0$ for all $k \geq 2$ where $\lambda_{k}(A)$ is the $k$ th largest eigenvalue of $A$ in terms of magnitude $\left|\lambda_{k}\right|$. Notice that $R=S Q S^{-1}$. Since $A S=S J$ the first column of $S$ is $w_{r}$. Similarly, $S^{-1} A=J S^{-1}$ that means the first row of $S^{-1}$ is $w_{l}^{T}$. Due to the fact that $S^{-1} S=I$, $w_{l}$ satisfies the property $w_{l}^{T} w_{r}=1$ as stated in the question. A straightforward calculation shows that $R=w_{r} w_{l}^{T} \in M_{n}$.

\section{Main Results}

In this section, we present three of our main results: i) characterization of all connected digraphs that solve average-consensus problem using Protocol (A), and ii) the relation between the performance of Protocol (A) and the Fiedler eigenvalue (i.e. algebraic connectivity) of graphs, and iii) robust agreement under switching information flows and link failures/creations.

\subsection{Balanced Graphs and Average-Consensus on Digraphs}

The following class of digraphs turns out to be instrumental in solving average-consensus problems:

Definition 1. (balanced graphs) We say the node $v_{i}$ of a digraph $G=(\mathcal{V}, \mathcal{E}, \mathcal{A})$ is balanced if and only if its in-degree and out-degree are equal, i.e. $\operatorname{deg}_{\text {out }}\left(v_{i}\right)=\operatorname{deg}_{\text {in }}\left(v_{i}\right)$. A graph $G=(\mathcal{V}, \mathcal{E}, \mathcal{A})$ is called balanced if and only if all of its nodes are balanced, i.e. $\sum_{j} a_{i j}=\sum_{j} a_{j i}, \forall i$.

Example 1. Any undirected graph is balanced. Furthermore, the digraphs shown in Figure 3 are all balanced.

Here is our first main result:

Theorem 2. Consider a network of integrators with directed information flow $G=(\mathcal{V}, \mathcal{E}, \mathcal{A})$ that is strongly connected. Then, $G$ globally asymptotically solves the average-consensus problem using Protocol (A) if and only if $G$ is balanced.

Proof. The proof follows from Propositions 3 and 4 .

Remark 2. According to Theorem 2, if a graph is not balanced, then it does not globally solve the average consensus-problem using Protocol (A). This assertion is consistent with the counterexample given in Figure 2. 


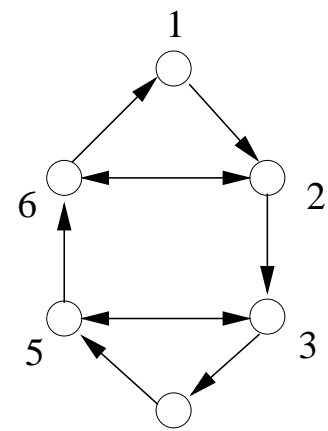

(a)

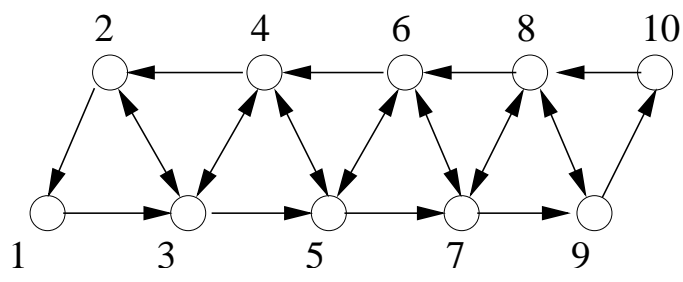

(b)

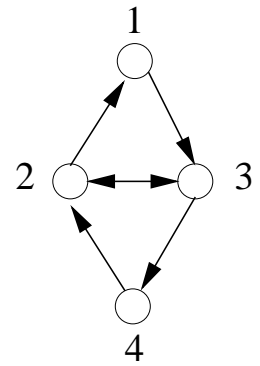

(c)

Figure 3: Three examples of balanced graphs.

Proposition 3. Consider a network of integrators with directed information flow $G=(\mathcal{V}, \mathcal{E}, \mathcal{A})$ that is strongly connected. Then, the digraph $G$ globally asymptotically solves the average-consensus problem using Protocol (A) if and only if $\mathbf{1}^{T} L=0$.

Proof. From Theorem 1, with $w_{r}=\frac{1}{\sqrt{n}} \mathbf{1}$ we obtain

$$
x^{*}=\lim _{t \rightarrow+\infty} x(t)=R x_{0}=w_{r}\left(w_{l}^{T} x_{0}\right)=\frac{1}{\sqrt{n}}\left(w_{l}^{T} x_{0}\right) \mathbf{1} .
$$

This implies Protocol 1 globally exponentially solves a consensus problem with the decision value $\frac{1}{\sqrt{n}}\left(w_{l}^{T} x_{0}\right)$ for each node. If this decision value is equal to $\operatorname{Ave}\left(x_{0}\right), \forall x_{0} \in \mathbb{R}^{n}$, then necessarily $\frac{1}{\sqrt{n}} w_{l}=\frac{1}{\sqrt{n}}$, i.e. $w_{l}=w_{r}=\frac{1}{\sqrt{n}} \mathbf{1}$. This implies that $\mathbf{1}$ is the left eigenvector of $L$. To prove the converse, assume that $\mathbf{1}^{T} L=0$. Let us take $w_{r}=\frac{1}{\sqrt{n}} \mathbf{1}, w_{l}=\beta \mathbf{1}$ with $\beta \in \mathbb{R}, \beta \neq 0$. From condition $w_{l}^{T} w_{r}=1$, we get $\beta=\frac{1}{\sqrt{n}}$ and $w_{l}=\frac{1}{\sqrt{n}} \mathbf{1}$. This means that the decision value for every node is $\frac{1}{\sqrt{n}}\left(w_{l}^{T} x_{0}\right)=\frac{1}{n} \mathbf{1}^{T} x_{0}=\operatorname{Ave}\left(x_{0}\right)$.

Corollary 1. Assume all the conditions in Proposition 3 hold. Suppose $L$ has a left eigenvector $\gamma=\left(\gamma_{1}, \ldots, \gamma_{n}\right)^{T}$ associated with $\lambda=0$ that is a nonnegative vector in $\mathbb{R}^{n}$ (i.e. a vector with non-negative elements) satisfying $\sum_{i} \gamma_{i}>0$. Then, the decision value of the group after reaching consensus is given by

$$
\alpha=\frac{\sum_{i} \gamma_{i} x_{i}(0)}{\sum_{i} \gamma_{i}}
$$

i.e. the decision value is in the convex hull of initial values of the nodes.

Proof. We have $\gamma^{T} L=0$ and thus $\gamma^{T} u \equiv 0$ (because $u=-L x$ ). Therefore, $\beta=\gamma^{T} x$ is an invariant quantity. Assume, the digraph $G$ is not balanced. Then, still an agreement is asymptotically reached. Let $\alpha$ be the decision value of all nodes after reaching consensus. We have $\gamma^{T} x^{*}=\gamma^{T} x(0)$ due to invariance of $\gamma^{T} x(t)$. But $x^{*}=\alpha \mathbf{1}$, thus we obtain

$$
\left(\sum_{i} \gamma_{i}\right) \alpha=\gamma^{T} x(0)
$$


and the result follows.

The following result shows that if one of the agents uses a relatively small update rate (or step-size), i.e. $\gamma_{i^{*}} \gg \gamma_{i}$ for all $i \neq i *$. Then, the value of all nodes converges to the value of $x_{i}^{*}$. In other words, the agent $i^{*}$ plays the role of a leader in leader-follower type architecture.

Corollary 2. (multi-rate integrators) Consider a network of multi-rate integrator with the node dynamics

$$
\gamma_{i} \dot{x}_{i}=u_{i}, \quad \gamma_{i}>0, \forall i \in \mathcal{I}
$$

Assume each node applies Protocol (A). Then, an agreement is globally asymptotically reached and the decision value of the group is

$$
\alpha=\frac{\sum_{i} \gamma_{i} x_{i}(0)}{\sum_{i} \gamma_{i}}
$$

Proof. The dynamics of the network evolves according to

$$
D \dot{x}=-L x
$$

where $D=\operatorname{diag}(\gamma)$ is a diagonal matrix with the $i$ th diagonal element that is equal to $\gamma_{i}>0$. The last equation can be rewritten as

$$
\dot{x}=-\tilde{L} x
$$

where $\tilde{L}=D^{-1} L=\operatorname{diag}\left(1 / \gamma_{1}, \ldots, 1 / \gamma_{n}\right) L$ is a valid Laplacian matrix for a digraph $\tilde{G}$ with adjacency matrix $\tilde{A}=D^{-1} \mathcal{A}$ (i.e. the weights of the edges leaving node $i$ are divided by $\gamma_{i}$ ). Clearly, $\gamma$ is a vector with positive elements that is the left eigenvector of $\tilde{L}$ and based on Corollary 1 the decision value is in the weighted average of $x_{i}(0)$ 's with weights specified by $\gamma$.

Proposition 4. Let $G=(\mathcal{V}, \mathcal{E}, \mathcal{A})$ be a digraph with an adjacency matrix $\mathcal{A}=\left[a_{i j}\right]$ satisfying $a_{i i}=0, \forall i$. Then, all the following statements are equivalent:

i) $G$ is balanced,

ii) $w_{l}=\mathbf{1}$ is the left eigenvector of the Laplacian of $G$ associates with the zero eigenvalue, i.e. $\mathbf{1}^{T} L=0$.

iii) $\sum_{i=1}^{n} u_{i}=0, \forall x \in \mathbb{R}^{n}$ with $u_{i}=\sum_{j \in N_{i}} a_{i j}\left(x_{j}-x_{i}\right)$.

Proof. We show $i) \Longleftrightarrow i i)$ and $i i) \Longleftrightarrow i i i)$.

Proof of $i) \Longleftrightarrow i i)$ : We have $\Delta_{i i}=\operatorname{deg}_{\text {out }}\left(v_{i}\right)$ and $\operatorname{deg}_{i n}\left(v_{i}\right)=\sum_{j, j \neq i} a_{j i}$, thus the $i$ th column sum of $L$ is equal to

$$
\sum_{i} l_{j i}=\sum_{i, j \neq i} l_{j i}+l_{i i}=-\operatorname{deg}_{i n}\left(v_{i}\right)+\operatorname{deg}_{\text {out }}\left(v_{i}\right)=0 \Leftrightarrow \text { node } v_{i} \text { of } G \text { is balanced }
$$

Noting that the $i$ column sum of $L$ is the same as the $i$ th element of the row vector $\mathbf{1}^{T} L$, one concludes that $\mathbf{1}^{T} L=0$ iff all the nodes of $G$ are balanced, i.e. $G$ is balanced. 0 .

Proof of $i i) \Longleftrightarrow i i i)$ : Since $u=-L x,\left(\sum_{i} u_{i}=0, \forall x\right) \Leftrightarrow\left(\mathbf{1}^{T} u=-\left(\mathbf{1}^{T} L\right) x=0, \forall x\right) \Leftrightarrow \mathbf{1}^{T} L=$

Remark 3. Notice that in Proposition 4, the graph $G$ does not need to be connected. Moreover, Proposition 4 holds for a general digraph with an weighted adjacency matrix that has nonzero diagonal elements. However, here we assume the no trivial cycles of length one in the graph. If $a_{i i} \neq 0$, then $i \in N_{i}$ and the term $a_{i i}\left(x_{i}-x_{i}\right) \equiv 0$. In other words, $u_{i}$ remains unchanged. 


\subsection{Performance of Group Agreement and the Mirror Operation}

In this section, we discuss performance issues of Protocol (A) with balanced graphs. An important consequence of Proposition 4 is that for networks with balanced information flow, $\alpha=$ Ave $(x)$ is an invariant quantity. This is certainly not true for an arbitrary digraph. The invariance of Ave $(x)$ allows decomposition of $x$ according to the following equation:

$$
x=\alpha \mathbf{1}+\delta
$$

where $\alpha=\operatorname{Ave}(x)$ and $\delta \in \mathbb{R}^{n}$ satisfies $\sum_{i} \delta_{i}=0$. We refer to $\delta$ as the (group) disagreement vector. The vector $\delta$ is orthogonal to 1 and belongs to an $(n-1)$-dimensional subspace called the disagreement eigenspace of $L$ provided that $G$ is strongly connected. Moreover, $\delta$ evolves according to the (group) disagreement dynamics given by

$$
\dot{\delta}=-L \delta
$$

It turns out that a useful property of balanced graphs is that for any balanced digraph $G$, there exists an undirected graph that has the same disagreement function as $G$. In the following, we formally define this induced undirected graph.

Definition 2. (mirror graph/operation) Let $G=(\mathcal{V}, \mathcal{E}, \mathcal{A})$ be weighted digraph. Let $\tilde{\mathcal{E}}$ be the set of reverse edges of $G$ obtained by reversing the order of all the pairs in $\mathcal{E}$. The mirror of $G$ denoted by $\hat{G}=\mathcal{M}(G)$ is an undirected graph in the form $\hat{G}=(\mathcal{V}, \hat{\mathcal{E}}, \hat{\mathcal{A}})$ with the same set of nodes as $G$, the set of edges $\hat{\mathcal{E}}=\mathcal{E} \cup \tilde{\mathcal{E}}$, and the symmetric adjacency matrix $\hat{\mathcal{A}}=\left[\hat{a}_{i j}\right]$ with elements

$$
\hat{a}_{i j}=\hat{a}_{j i}=\frac{a_{i j}+a_{j i}}{2} \geq 0
$$

The following result shows that the operations of $\mathcal{L}$ and $S y m$ on a weighted adjacency matrix $\mathcal{A}$ commute. Moreover,

Theorem 3. Let $G$ be a digraph with adjacency matrix $\mathcal{A}=\operatorname{adj}(G)$ and Laplacian $L=\mathcal{L}(G)$. Then $L_{s}=\operatorname{Sym}(L)=\left(L+L^{T}\right) / 2$ is a valid Laplacian matrix for $\hat{G}=\mathcal{M}(G)$ if and only if $G$ is balanced, i.e. the following diagram commutes iff $G$ is balanced

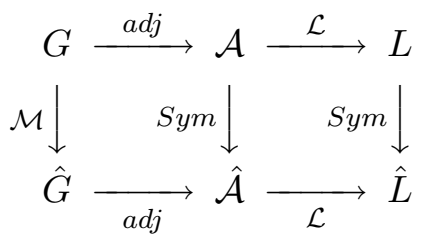

Moreover, if $G$ is balanced, the disagreement functions of $G$ and $\hat{G}$ are equal.

Proof. We know that $G$ is balanced iff $\mathbf{1}^{T} L=0$. Since $L \mathbf{1}=0$, we have $\mathbf{1}^{T} L=0 \Longleftrightarrow$ $\frac{1}{2}\left(L+L^{T}\right) \mathbf{1}=0$. Thus, $G$ is balanced iff $L_{s}$ has a right eigenvector of $\mathbf{1}$ associated with $\lambda=0$, i.e. $L_{s}$ is a valid Laplacian matrix. Now, we prove that $L_{s}=\mathcal{L}(\hat{G})$. For doing so, let us calculate $\hat{\Delta}$ element-wise, we get

$$
\hat{\Delta}_{i i}=\sum_{j} \frac{a_{i j}+a_{j i}}{2}=\frac{1}{2}\left(\operatorname{deg}_{\text {out }}\left(v_{i}\right)+\operatorname{deg}_{\text {in }}\left(v_{i}\right)\right)=\operatorname{deg}_{\text {out }}\left(v_{i}\right)=\Delta_{i i}
$$


Thus, $\hat{\Delta}=\Delta$. On the other hand, we have

$$
L_{s}=\frac{1}{2}\left(L+L^{T}\right)=\Delta-\frac{A+A^{T}}{2}=\hat{\Delta}-\hat{A}=\hat{L}=\mathcal{L}(\hat{G})
$$

The last part simply follows from the fact that $\hat{L}$ is equal to the symmetric part of $L$ and $x^{T}(L-$ $\left.L^{T}\right) x \equiv 0$.

Notation. For simplicity of notation, in the context of algebraic graph theory, $\lambda_{k}(G)$ is used to denote $\lambda_{k}(\mathcal{L}(G))$.

Now, we are ready to present our main result on performance of the Protocol (A) in terms of the speed of reaching a consensus as a group.

Theorem 4. (performance of agreement) Consider a network of integrators with a directed information flow $G$ that is balanced and strongly connected. Then, given Protocol (A), the following statements hold:

i) the group disagreement (vector) $\delta$ as the solution of the disagreement dynamics in (16) globally asymptotically vanishes with a speed that is equal to $\kappa=\lambda_{2}(\hat{G})$ (or the Fiedler eigenvalue of the mirror graph of $G$ ), i.e.

$$
\|\delta(t)\| \leq\|\delta(0)\| \exp (-\kappa t)
$$

ii) the following smooth, positive definite, and proper function

$$
V(\delta)=\frac{1}{2}\|\delta\|^{2}
$$

is a valid Lyapunov function for the disagreement dynamics.

Proof. We have

$$
\dot{V}=-\delta^{T} L \delta=-\delta^{T} L_{s} \delta=-\delta^{T} \hat{L} \delta \leq-\lambda_{2}(\hat{G})\|\delta\|^{2}=-2 \kappa V(\delta)<0, \forall \delta \neq 0
$$

This proves that $V(\delta)$ is a valid Lyapunov function for the group disagreement dynamics. Moreover, $\delta(t)$ vanishes globally exponentially fast with a speed of $\kappa$ as $t \rightarrow+\infty$. The fact that $L_{s}=\hat{L}$ is a valid Laplacian matrix for an undirected graph (i.e. mirror of $G$ ) follows from Theorem 3 and the inequality

$$
\delta^{T} \hat{L} \delta \geq \lambda_{2}(\hat{G})\|\delta\|^{2}, \quad \forall \delta: \mathbf{1}^{T} \delta=0
$$

which is due to equation (5).

A well-known observation regarding the Fiedler eigenvalue of an undirected graph is that for dense graphs $\lambda_{2}$ is relatively large and for sparse graphs $\lambda_{2}$ is relatively small [8] (this is why $\lambda_{2}$ is called the algebraic connectivity). According to this observation, from Theorem 4, one can conclude that a network with dense interconnections solves an agreement problem faster than a connected but sparse network. This is consistent with common sense regarding agreement in a group. As a special case, a cycle of length $n$ that creates a balanced digraph on $n$ nodes solves an agreement problem. However, this is a relatively slow way to solve such a consensus problem. 


\subsection{Agreement in Networks with Switching Topology}

Consider a network of mobile agents that communicate with each other and need to agree upon a certain objective of interest or perform synchronization. Since, the nodes of the network are moving, it is not hard to imagine that some of the existing communication links can fail simply due to the existence of an obstacle between two agents. The opposite situation can arise where new links between nearby agents are craeted because the agents come to an effective range of detection with respect to each other. In other words, in the graph $G$ representing the information flow of the network, certain edges can be added or removed from $G$. Here, we are interested to investigate that in case of a network with switching topology whether it is still possible to reach a consensus or not.

Consider a hybrid system with a continuous-state $x \in \mathbb{R}^{n}$ and a discrete-state $G$ that belongs to a finite set of digraphs

$$
\Gamma_{n}=\{G: G \text { is a digraph of order } n \text { that is strongly connected and balanced }\}
$$

that can be analytically expressed in the form

$$
\Gamma_{n}=\left\{G=(\mathcal{V}, \mathcal{E}, \mathcal{A}): \operatorname{rank}(\mathcal{L}(G))=n-1, \mathbf{1}^{T} \mathcal{L}(G)=0\right\} .
$$

Given the node dynamics and protocol, the continuous-state of the system evolves according to the following dynamics

$$
\dot{x}(t)=-\mathcal{L}\left(G_{k}\right) x(t), \quad k=s(t), G_{k} \in \Gamma_{n}
$$

where $s(t): \mathbb{R}_{\geq 0} \rightarrow \mathcal{I}_{\Gamma_{n}}$ is a switching signal and $\mathcal{I}_{\Gamma_{n}} \subset \mathbb{N}$ is the index set associated with the elements of $\Gamma_{n}$. Clearly, $\Gamma_{n}$ is a finite set, because either a digraph has no edges or it is a complete graph with $n(n-1)$ directed edges.

The key in solving the agreement problem for mobile networks with switching topology is a basic property of the Lyapunov function in (20) and the properties of balanced graphs. Note that the function $V(\delta)=\frac{1}{2}\|\delta\|^{2}$ does not depend on $G$ or $L=\mathcal{L}(G)$. This property of $V(\delta)$ makes it an appropriate candidate as a common Lyapunov function for stability analysis of the switching system (24).

Theorem 5. For any arbitrary switching signal $s(\cdot)$, the solution of the switching system (24), globally asymptotically converges to Ave $(x(0))$ (i.e. average-consensus is reached). Moreover, the following smooth, positive definite, and proper function

$$
V(\delta)=\frac{1}{2}\|\delta\|^{2}
$$

is a valid common Lyapunov function for the disagreement dynamics given by

$$
\dot{\delta}(t)=-\mathcal{L}\left(G_{k}\right) \delta(t), \quad k=s(t), G_{k} \in \Gamma_{n}
$$

Furthermore, the disagreement vector $\delta$ vanishes exponentially fast with the least rate of

$$
\left.\kappa^{*}=\min _{G \in \Gamma_{n}} \lambda_{2}(\mathcal{L}(\hat{G}))\right)
$$

In other words, $\|\delta(t)\| \leq\|\delta(0)\| \exp \left(-\kappa^{*} t\right)$. 
Proof. Due the fact that $G_{k}$ is balanced for all $k$ and $u=-\mathcal{L}\left(G_{k}\right) x$, we have $\mathbf{1}^{T} u=-\left(\mathbf{1}^{T} \mathcal{L}\left(G_{k}\right)\right) x \equiv$ 0 . Thus, $\alpha=\operatorname{Ave}(x)$ is an invariant quantity which allows us to decompose $x$ as $x=\alpha \mathbf{1}+\delta$. Therefore, the disagreement switching system induced by (24) takes the form (26). Calculating $\dot{V}$, we get

$$
\dot{V}=-\delta^{T} \mathcal{L}\left(G_{k}\right) \delta=-\delta^{T} \mathcal{L}\left(\hat{G}_{k}\right) \delta \leq-\lambda_{2}\left(\mathcal{L}\left(\hat{G}_{k}\right)\right)\|\delta\|^{2} \leq-\kappa^{*}\|\delta\|^{2}=-2 \kappa^{*} V(\delta)<0, \forall \delta \neq 0
$$

This guarantees that $V(\delta)$ is a valid common Lyapunov function for the disagreement switching system (26). Moreover, we have

$$
V(\delta(t)) \leq V(\delta(0)) \exp \left(-2 \kappa^{*} t\right) \Rightarrow\|\delta(t)\| \leq\|\delta(0)\| \exp \left(-\kappa^{*} t\right)
$$

and the disagreement vector $\delta(t)$ globally exponentially vanishes with a speed of $\kappa^{*}>0$ as $t \rightarrow+\infty$. Finally, the minimum in (27) always exists and is achieved because $\Gamma_{n}$ is a finite set.

\section{Simulation Results}

Figure 4 shows four different networks each with $n=10$ nodes that are all strongly connected and balanced. The weights associated with all the edges are 1. For the following initial node values satisfying Ave $(x(0))=0$

$$
x(0)=(-10.2999,0.2575,-4.4997,3.6258,3.0922,9.0156,3.5099,-2.6645,2.4552,-4.4921)^{T}
$$

we have plotted the state trajectories and the disagreement function $\|\delta\|^{2}$ associated with these four digraphs in Figure 5. It is clear that as the number of the edges of the graph increase, algebraic connectivity (or $\lambda_{2}$ ) increases, and the settling time of the trajectory of the node values decreases. The case of a directed cycle of length $n=10$, or $G_{a}$, has the largest over-shoot. In all four cases, an agreement is asymptotically reached and the performance is improved as a function of $\lambda_{2}\left(\hat{G}_{k}\right)$ for $k \in\{a, b, c, d\}$.
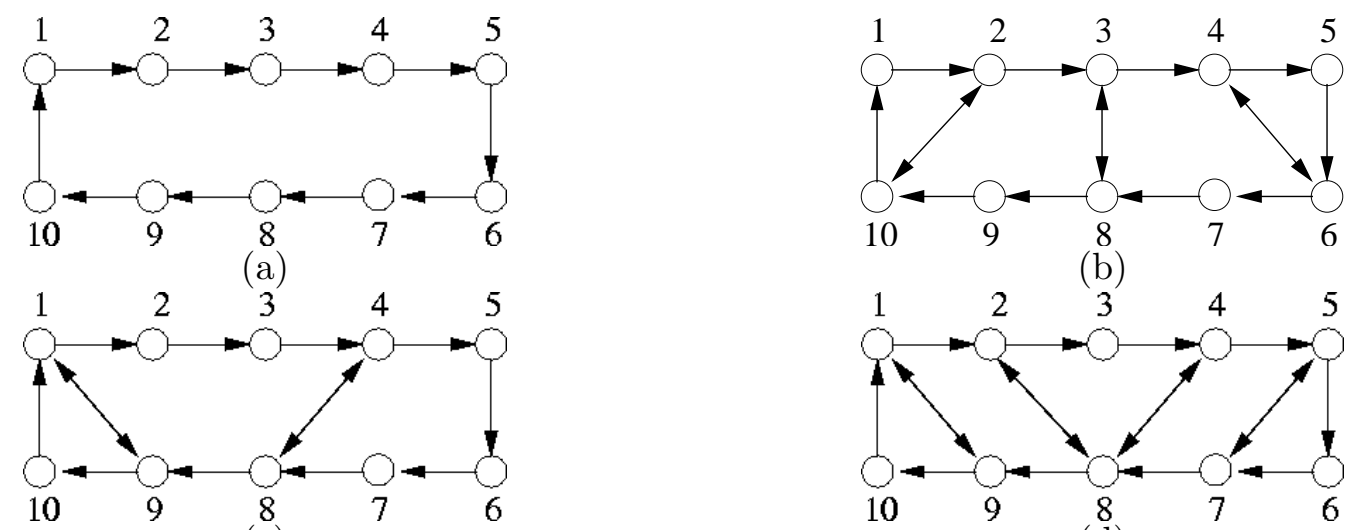

(c)

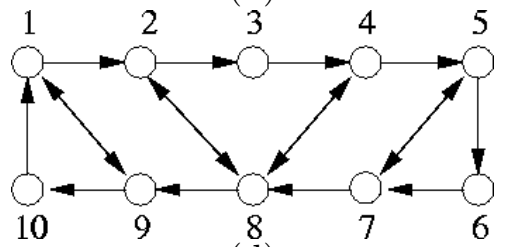

(d)

Figure 4: For examples of balanced and strongly connected digraphs: (a) $G_{a}$, (b) $G_{b}$, (c) $G_{c}$, and (d) $G_{d}$ satisfying. 

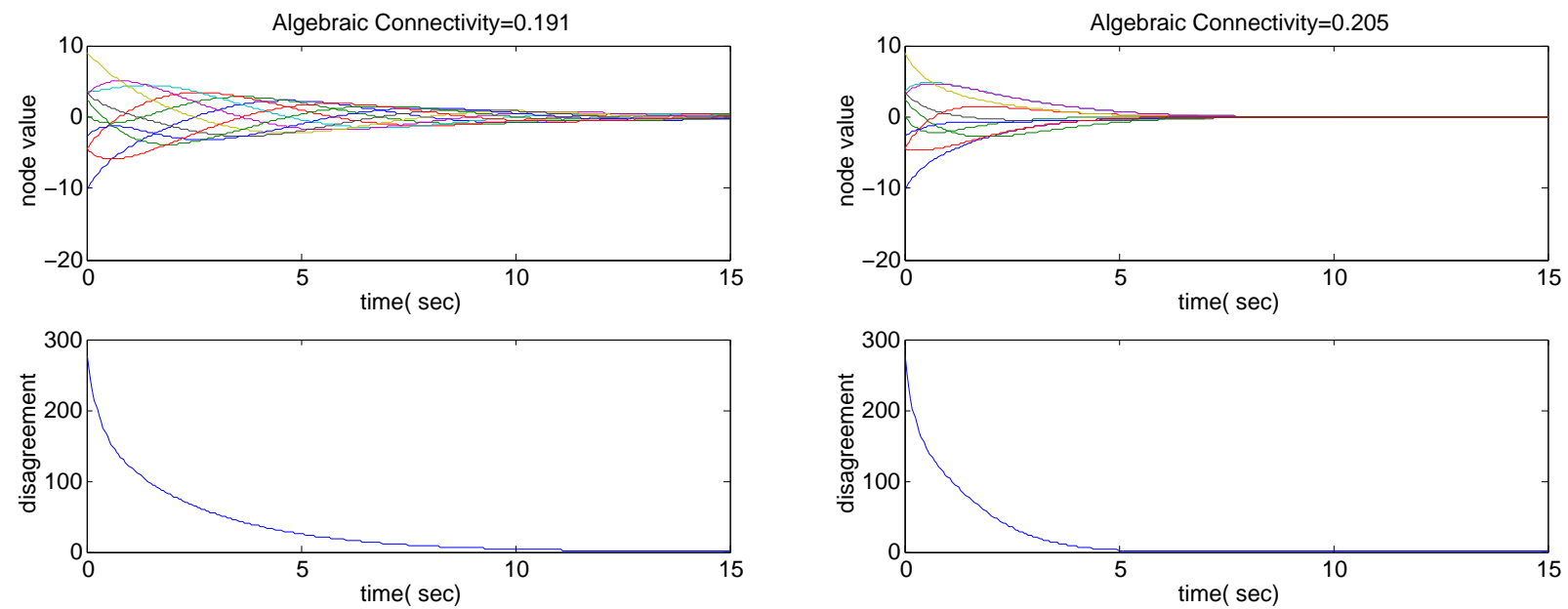

(a)

(b)
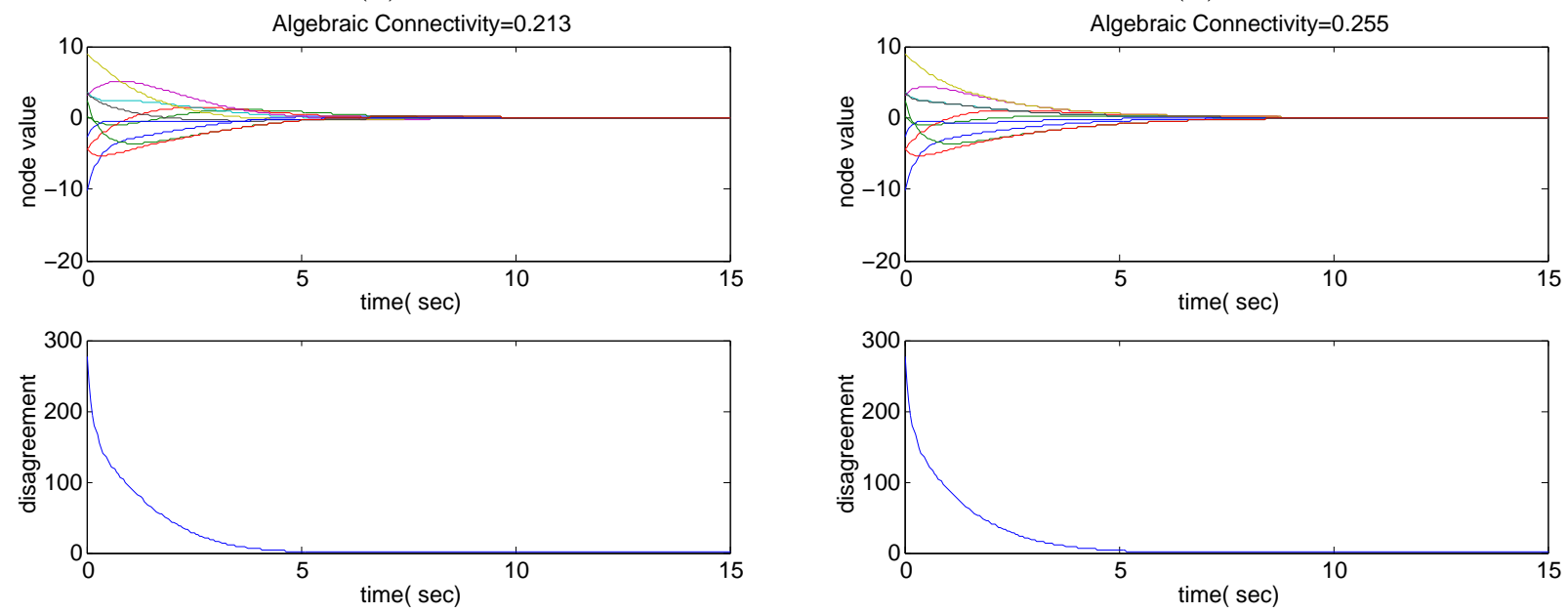

(c)

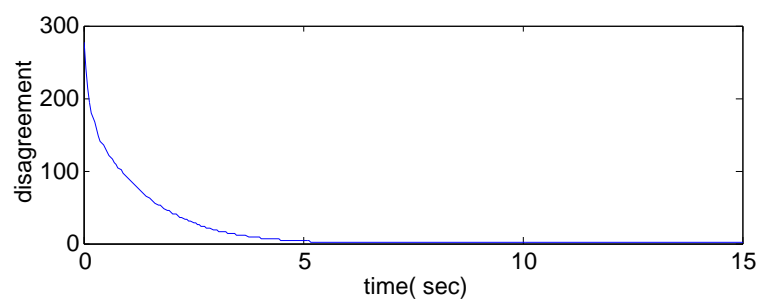

(d)

Figure 5: For examples of balanced and strongly connected digraphs: (a) $G_{a}$, (b) $G_{b}$, (c) $G_{c}$, and (d) $G_{d}$ satisfying.

In Figure 6(a), a finite-state machine is shown with the set of states $\left\{G_{a}, G_{b}, G_{c}, G_{d}\right\}$ representing the discrete-states of a network with switching topology as a hybrid system. The hybrid system starts at the discrete-state $G_{b}$ and switches every $T=1$ second to the next state according to the state machine in Figure 6(a). The continuous-time state trajectories and the group disagreement (i.e. $\|\delta\|^{2}$ ) of the network are shown in Figure 6(b). Clearly, the group disagreement is monotonically decreasing. One can observe that an average-consensus is reached asymptotically. Moreover, the group disagreement vanishes exponentially fast.

\section{Conclusion}

In this paper, we addressed convergence and performance problems for an agreement protocol for a network of dynamic agents with integrator dynamics and directed information flow. Moreover, we 


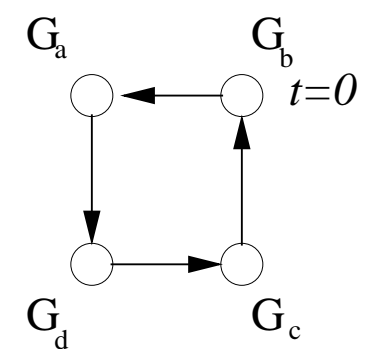

(a)
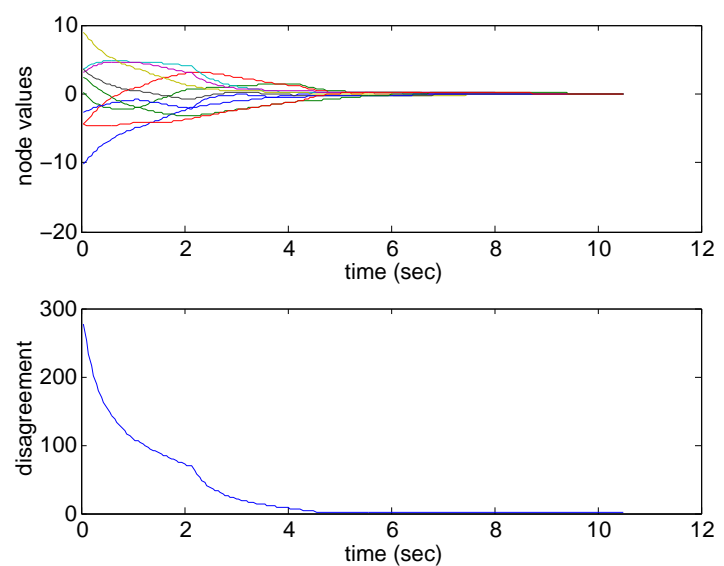

(b)

Figure 6: (a) A finite-state machine with four states representing the discrete-states of a network with variable topology and (b) trajectory of the node values and the group disagreement for a network with a switching information flow.

analyzed robustness of this consensus protocol changes in the topology of a network with mobile nodes. We showed that balanced graphs are the only type of digraphs that solve the averageconsensus problem with the aforementioned agreement protocol. Moreover, we proved that for any balanced digraph, there exists an undirected graph called the mirror graph. This mirror graph has the same Laplacian disagreement function as the original digraph and its Laplacian is the symmetric part of the Laplacian of the network in question. A commutative diagram was given that shows the operations of taking Laplacian and symmetric part of a matrix commute for weighted adjacency matrices of balanced graphs.

Fiedler eigenvalue of the mirror of a digraph is used to quantify the speed of convergence of the above agreement protocol. Moreover, a simple disagreement function was introduced as a Lyapunov function for the group disagreement dynamics. This was later used to provide a common Lyapunov function that allowed convergence analysis of an agreement protocol for a network with mobile nodes and switching topology. We provided simulation results that were consistent with our theoretical predictions and demonstrated the use of new graph theoretical tools and notions.

\section{Acknowledgements}

This research is supported in part by AFOSR under the grant F49620-01-1-0361 and by DARPA under the grant F33615-98-C-3613.

\section{References}

[1] N. Biggs. Algebraic Graph Theory. Cambridge Tracks in Mathematics. Cambridge University Press, 1974. 
[2] J. P. Desai, J. P. Ostrowski, and V. Kumar. Modeling and control of formations of nonholonomic mobile robots. IEEE Trans. on Robotics and Automation, 17(6), December 2002.

[3] R. Diestel. Graph Theory, volume 173 of Graduate Texts in Mathematics. Springer-Verlag, 2000 .

[4] A. Fax and R. M. Murray. Graph laplacians and stabilization of vehicle formations. The 15th IFAC World Congress, June 2002.

[5] A. Fax and R. M. Murray. Information Flow and Cooperative Control of Vehicle Formations. The 15th IFAC World Congress, June 2002.

[6] M. Fiedler. Algebraic connectivity of graphs. Czechoslovak Mathematical Journal, 23(98):298305, 1973.

[7] M. Fiedler. A property of eigenvectors of nonnegative symmetric matrices and its application to graph theory. Czechoslovak Mathematical Journal, 25(100):619-633, 1975.

[8] C. Godsil and G. Royle. Algebraic Graph Theory, volume 207 of Graduate Texts in Mathematics. Springer, 2001.

[9] R. A. Horn and C. R. Johnson. Matrix Analysis. Cambridge University Press, 1987.

[10] A. Jadbabaie, J. Lin, and S. A. Morse. Coordination of groups of mobile agents using nearest neighbor rules. IEEE Trans. on Automatic Control (to appear).

[11] R. W. Lawton, J. R. T. Beard and B. J. Young. A Decentralized Approach to Formation Maneuvers. IEEE Trans. on Robotics and Automation (to appear).

[12] N. A. Lynch. Distributed Algorithms. Morgan Kaufmann Publishers, Inc., 1997.

[13] M. Mesbahi and F. Y. Hadegh. Formation flying of multiple spacecraft via graphs, matrix inequalities, and switching. AIAA Journal of Guidance, Control, and Dynamics, 24(2):369377, March 2000.

[14] R. Olfati Saber and R. M. Murray. Consensus Protocols for Networks of Dynamic Agents. Proc. of the American Control Conference, June 2003.

[15] F. Paganini, J. Doyle, and S Low. Scalable laws for stable network congestion control. Proc. of the Int. Conf. on Decision and Control, Orlando, FL, Dec. 2001.

[16] C. W. Reynolds. Flocks, herds, and schools: a distributed behavioral model. Computer Graphics (ACM SIGGRAPH'87 Conference Proceedings), 21(4):25-34, July 1987.

[17] J. Toner and Y. Tu. Flocks, herds, and schools: A quantitative theory of flocking. Physical Review E, 58(4):4828-4858, October 1998.

[18] T. Vicsek, A. Cziroók, E. Ben-Jacob, and O. Cohen, I. Shochet. Novel type of phase transition in a system of self-deriven particles. Physical Review Letters, 75(6):1226-1229, August, 1995.

[19] H. Yamaguchi, T. Arai, and G. Beni. A distributed control scheme for multiple robotic vehicles to make group formations. Robotics and Autonomous Systems, 36:125-147, 2001. 